\title{
Myotonic dystrophies as a brain disorder
}

\author{
Giovanni Meola
}

Received: 29 April 2009/Accepted: 2 September 2010/Published online: 6 October 2010

(C) Springer-Verlag 2010

In their article, Romeo et al. [1] emphasize that abnormalities in blood perfusion and/or glucose metabolism are frequent in myotonic dystrophy type 1 (DM1 or Steinert's disease).

I would suggest that these abnormalities and others encompass all myotonic dystrophies. Myotonic dystrophies are autosomal dominant multisystem disorders. These include myotonic dystrophy type 1 (Steinert's disease or DM1) due to an abnormal CTG expansion on chromosome 19 coding for protein kinase DMPK and myotonic dystrophy type 2 (proximal myotonic myopathy, PROMM or DM2), due to an abnormal CCTG expansion on chromosome 3 coding for a zinc finger protein ZNF9. These abnormal nucleotide expansions cause an abnormal accumulation of mRNA which is sequestered within the nucleus by specific binding proteins thus altering mRNA splicing of proteins involved in the disease process [2].

There are clinical, neuropsychological and neuroimaging data that support the hypothesis of central nervous system involvement also in DM2. The typical cognitive and behavioural abnormalities in DM2 are like those of the classical adult form of DM1 but in DM2 these abnormalities are less severe. Patients with DM2 have a frontal dysexecutive syndrome characterized by apathy, reduced initiative and strategic abilities. Yet global cognitive performances are normal as demonstrated by normal IQ and MMSE test scores. Neuropsychological test have demonstrated a selective involvement of visual spatial functions which do not seem to correlate with hyperintense lesions demonstrated on traditional brain MRI sequences or to the degree

G. Meola $(\bowtie)$

IRCCS Policlinico San Donato, University of Milan,

Via Morandi, 30, 20097 San Donato Mil. (Milan), Italy

e-mail: giovanni.meola@unimi.it of cortical atrophy in the frontal and temporal poles, but indicate a selective frontal lobe involvement and a reduction of cerebral blood flow in these regions as documented by H20 PET studies [3-5]. From a behavioural point of view, the patients DM1 and DM2 have an avoidant personality trait, more rarely a passive personality trait [6]. These personality traits imply the patients tend to avoid regular outpatient visits, minimize their muscle symptoms and rarely seek medical attention. It is rather the unaffected family members who will notice difficulties during walking, stumbling and tripping over with an increase in frequency. A distinctive element between DM1 and DM2 is the absence of a congenital form in DM2 and the excessive daytime sleepiness in DM1. Extrapyramidal features like camptocormia have been described in some patients with DM2 and the dopaminergic pathway does not seem to justify extrapyramidal signs and symptoms in these patients [7].

In spite of the findings described above, advances in the study of DM1 and DM2 nervous system require development of better-designed study models. For instance, modelling of transgenic mice expressing CUG expansion RNA under the control of a strong neuronal-specific promoter, will allow the exacerbation of DM1 and DM2 neuronal manifestations. Such mouse models could serve as a basis for complete studies of DM1 and DM2 nervous system including brain anatomy analysis, behaviour and memory test, and molecular and cellular biology approach using brain samples and neuron cell cultures.

\section{References}

1. Romeo V, Pegoraro E, Squarzanti F et al (2010) Retrospective study on PET-SPECT imaging in a large cohort of myotonic 
dystrophy type 1 patients. Neurol Sci. doi:10.1007/s10072-0100406-2

2. Wheeler TM, Thornton CA (2007) Myotonic dystrophy: RNAmediated muscle disease. Curr Opin Neurol 20:572-576

3. Meola G, Sansone V, Perani D et al (1999) Reduced cerebral blood flow and impaired visual-spatial function in proximal myotonic myopathy. Neurology 53:1042-1050

4. Meola G, Sansone V (2007) Cerebral involvement in myotonic dystrophies. Muscle Nerve 36:294-306

5. Sansone V, Gandossini S, Cotelli M et al (2007) Cognitive impairment in adult myotonic dystrophies: a longitudinal study. Neurol Sci 28:9-15
6. Meola G, Sansone V, Perani D et al (2003) Executive dysfunction and avoidant personality trait in myotonic dystrophy type 1 (DM-1) and in proximal myotonic myopathy (PROMM/DM-2). Neuromuscul Disord 13:813-821

7. Sansone V, Meola G, Perani D (2006) Glucose metabolism and dopamine PET correlates in a patient with myotonic dystrophy type 2 and parkinsonism. J Neurol Neurosurg Psychiatry 77:425-426 